\title{
Steroid-Responsive Nephrotic Syndrome and Bilateral Renal Artery Stenosis: A Possible Role for Angiotensin-Mediated Podocyte Injury
}

\author{
Rahul Bhardwaj Imran Dosani Barbara A. Clark \\ Division of Nephrology and Hypertension, Allegheny General Hospital, \\ Pittsburgh, Pa., USA
}

\section{Key Words}

Nephrosis · Renal artery stenosis · Hypertension · Proteinuria

\begin{abstract}
Nephrotic syndrome (NS) associated with renal artery stenosis is not widely recognized or investigated as a cause of the NS. The mechanisms are incompletely understood, but have largely focused on hemodynamic factors resulting in hyperfiltration injury-induced focal and segmental glomerulosclerosis (FSGS) in the nonstenosed kidney with sparing of the stenotic kidney protected from hemodynamic stress. However, separation of hemodynamic from circulating factors (such as angiotensin II) as the cause of the nephrosis remains difficult. We report a patient presenting with NS who was incidentally discovered to have high-grade bilateral renal artery stenosis from fibromuscular dysplasia. Kidney biopsy revealed FSGS. Proteinuria in our patient did not initially respond to angiotensin-converting enzyme inhibition (ACEI) and correction of stenoses with angioplasties. There was prompt response to steroid treatment. A brief relapse several months later (without associated hypertension) responded to ACEI alone. This is the first reported case of an association between fibromuscular dysplasia and steroid-responsive nephrotic syndrome due to FSGS. This may shed insight into the nature of podocyte injury in patients with high angiotensin states and suggest a possible role for activated renin-angiotensin-aldosterone system (RAAS) triggering an immune-mediated injury, rather than hemodynamic insult. Furthermore the lack of initial response to angioplasty and ACEI suggests that RAAS-activated injury may in some cases require more aggressive immune modulatory therapy with steroids over and above angiotensin inhibition alone. This case also highlights the importance of being aware of possible occult renovascular disease contributing to idiopathic NS with FSGS even when hypertension is only modest.
\end{abstract}




\section{Introduction}

Renovascular hypertension with its associated activation of the renin-angiotensinaldosterone system (RAAS) has been reported under certain circumstances, to be associated with nephrotic syndrome (NS) [1-12]. This has most often been in cases of unilateral stenosis [1-5]. The mechanism is not fully elucidated but some authors have postulated that unilateral renal artery stenosis (RAS) produces hyperfiltration injury and secondary focal and segmental glomerulosclerosis (FSGS) in the unaffected kidney with demonstration of protection of the stenotic kidney from hemodynamic injury [1$5,12]$. This, however, could not account for the cases of NS where bilateral RAS is present $[10,11]$. Furthermore, there are several cases of unilateral stenosis where nephrectomy specimen of the stenotic kidney revealed FSGS $[6,8,9]$. These findings suggest that circulating factors related to RAAS activation may play a primary role. In most cases, the NS resolved either with revascularization, nephrectomy of the stenotic kidney or angiotensin blockade [1-11]. We are reporting a case presenting for evaluation of NS that was incidentally discovered to have bilateral RAS due to fibromuscular dysplasia (FMD). Renal biopsy revealed FSGS. The NS did not respond to revascularization or angiotensin blockade after a period of 4 weeks observation, but did respond promptly to steroids. A brief relapse, several months later without any associated hypertension, did respond to angiotensin-converting enzyme inhibitor (ACEI) alone. To our knowledge, ours is the first reported case that required steroids for remission. This might suggest that the RAAS system is in part an immune modulator in such cases of NS perhaps as important mechanistically as the generally accepted hypothesis of hemodynamically mediated hyperfiltration injury.

\section{Case Report}

A 49-year-old Caucasian female with past medical history of borderline hypertension, controlled by diet and exercise, presented to office for evaluation of ankle swelling, abdominal fullness, bloating, foamy urine and weight gain of 10 pounds over 3 months. There was no history of smoking, substance abuse or urinary tract infection. Exam showed blood pressure of 160/98, 2+ edema up to the knees bilaterally and epigastric bruit radiating to the flanks. Urinalysis showed 3+ proteinuria, 3+ blood, oval fat bodies and non-dysmorphic RBCs without any casts. Laboratory evaluation showed BUN 21 $\mathrm{mg} / \mathrm{dl}$, creatinine $1.04 \mathrm{mg} / \mathrm{dl}$, albumin $2.5 \mathrm{mg} / \mathrm{dl}$, cholesterol $459 \mathrm{mg} / \mathrm{dl}$ and LDL $325 \mathrm{mg} / \mathrm{dl}$. Random urine protein-to-creatinine ratio was $9.8 \mathrm{~g} / \mathrm{g}$. Complement levels ANA, ANCA, ASO titer, hepatitis B, C and HIV serologies were all negative. Renal artery Doppler ultrasound study showed high-grade leftsided stenosis. Renal biopsy showed glomeruli with few segmental areas of inflammation which had deposition of C3, and IgG. Electron microscopy showed diffuse foot processes fusion. No tubuloreticular structures were seen. Renal biopsy diagnosis was focal segmental glomerulosclerosis with cellular variant (fig. 1). Subsequent renal arteriogram was significant for severe bilateral FMD greater than $80 \%$ (fig. 2 ). She was sent for angioplasty of each of the renal arteries 2 weeks apart with reduction to less than $20 \%$. Four weeks after the last angioplasty, her random urine protein-tocreatinine ratio remained elevated at $9.3 \mathrm{~g} / \mathrm{g}$ despite adequate blood pressure control with lisinopril. She was then treated with prednisone $80 \mathrm{mg}$ every other day. Within 3 weeks of steroid therapy, there was significant improvement with resolution of proteinuria $(0.2 \mathrm{~g} / \mathrm{g})$ and dyslipidemia (cholesterol 253). Steroids were tapered off over the next 4 weeks. Sixteen weeks later urine protein remained at $0.2 \mathrm{~g} / \mathrm{g}$ with use of lisinopril $10 \mathrm{mg}$ daily. With normotension, the lisniopril was then discontinued. However, 4 weeks after discontinuation of the ACEI, despite normal blood pressure, she developed a relapse with edema and random protein-to-creatinine ratio of $4 \mathrm{~g} / \mathrm{g}$. This time reinstitution of lisinopril resulted in prompt remission within 1 week. She has subsequently had normotension and no proteinuria maintained on low-dose ACEI for 2 years. 


\section{Discussion}

In existing medical literature there have been a few well-documented cases of unilateral RAS associated with NS [1-6]. The mechanism has been postulated to be hemodynamic (hyperfiltration) injury to the nonstenosed kidney with protection from this injury in the stenosed kidney. Most of these cases responded with resolution of nephrotic-range proteinuria after correction of stenosis, removal of the stenotic kidney or with inhibition of angiotensin [1-11]. However, there are also a few cases of NS with bilateral RAS and a few cases of FSGS in biopsy of the stenosed kidney [6-11].These cases suggest that the etiology of the protein loss is more than hemodyamically mediated podocyte injury and raises an alternative explanation for the activated RAAS in podocyte injury. It seems likely that both circulating factors (angiotensin and/or aldosterone) and hemodynamic factors are important. To our knowledge, ours is first case of NS (from FSGS) with simultaneous discovery of bilateral renal artery stenosis (due to FMD) that required steroid use for remission. Both angiotensin II and aldosterone have pleiotropic effects, which could also contribute to proteinuria [1315]. Angiotensin II can act as a local growth factor with proliferative, profibrogenic and proinflammatory properties. In rats, infusion of angiotensin II has been shown to produce reversible nephrotic-range proteinuria [14]. Mechanisms through which angiotensin II can contribute to proteinuria are not completely understood, though possibilities include direct action on vascular endothelial cells causing widened intercellular space, alteration of sialoglycoprotein concentration and anionic charge on basement membrane [13-15]. Rossignol et al. [16] reported that the degree of proteinuria in patients with RAS is directly related to active renin concentration (and hence angiotensin levels) irrespective of blood pressure. Immunosuppressive treatment has not been recommended in the treatment of NS from FSGS in such patients. In our patient, FSGS causing NS was noted in association with bilateral renal artery stenosis from FMD. Though hypertension itself can cause modest proteinuria, it is generally in the non-nephrotic range. Occasionally accelerated hypertension and hypertensive crises can cause nephrotic-range proteinuria [3], but this did not seem a possibility in this case as the rise in blood pressure was only modest and nonsustained. FSGS is a pathological diagnosis and can be secondary to various pathophysiologic mechanisms. In primary FSGS there is an immunologic injury to the podocyte with a proposed circulating permeability factor [17]. FSGS can also be secondary to reduced nephron mass from conditions like low birth weight, unilateral renal agenesis, surgery, trauma, or reflux nephropathy [18]. It can also be seen in association with patients with lymphomas, sickle-cell disease, obesity or cyanotic congenital heart disease $[19,20]$. Other causes include HIV, hepatitis B, and heroin abuse $[21,22]$. Certain medications including pamidronate and lithium can also be associated with secondary FSGS [23]. There are even recent case reports of FSGS secondary to anabolic steroid use, raising the role of sex hormones in podocyte function and on predisposition to hemodynamic stress [24]. Animal studies suggest a protective role of estrogen on podocyte function and estrogens may provide a protective role against other glomerular disorders, such as diabetic nephropathy that is lost after menopause [15]. Although our patient was perimenopausal, her clinical background and relevant investigations ruled out any secondary cause of FSGS other than RAS. It is interesting also to speculate on the role of the perimenopausal state in our patient and whether declining level of estrogens (with their proposed protective effect) may have 
uniquely increased her susceptibility to podocyte injury in this setting. Nonresponse of proteinuria to treatment with ACEI and correction of stenosis with angioplasty probably excludes hyperfiltration injury as the sole cause in our patient. However, we speculate that the activated RAAS system triggered diffuse podocyte injury that did not immediately respond to correction of the activated state with either angioplasty or ACEI. Our patient had a rapid response to steroids suggesting additional 'downstream' immunogenic processes following RAAS activation that may require more than just RAAS inhibition to correct once triggered. Of interest, a relapse, when detected and treated quickly with ACEI alone, did respond, suggesting that perhaps this immunogenic cascade can be interrupted with angiotensin inhibition alone if caught early enough in the disease state. It is possssible that this case may represent the coincidental concomitance of steroid-responsive primary FSGS associated with unrelated bilateral RAS. However, the relapse responding to ACE inhibition supports our hypothesis. It should also be noted that the degree of activation of the RAAS is uncertain in our case as no renin/angiotensin/aldosterone measurements were made to document changes before and after angioplasty.

In summary, we report the first case of simultaneous presentation of steroidresponsive NS and bilateral renal artery stenosis due to FMD. The NS did not initially respond to ACEI therapy or correction of stenosis with angioplasty, but did respond quickly to steroid therapy. We speculate about the role of an activated RAAS in triggering a cascade of podocyte injury leading to an immune-modulated FSGS that may require steroid use if reversal of the RAAS state does not occur early in the course of the disease state. Thadhani et al. [9] reported that a third of patients over the age of 50 with FSGS had renal vascular disease. This highlights the importance of being aware of the possibility of underlying renal artery stenosis in cases of otherwise unexplained FSGS (even steroid-responsive FSGS).

\section{Disclosure Statement}

There are no conflicts of interest to report. 


\section{Case Reports in Nephrology and Urology}
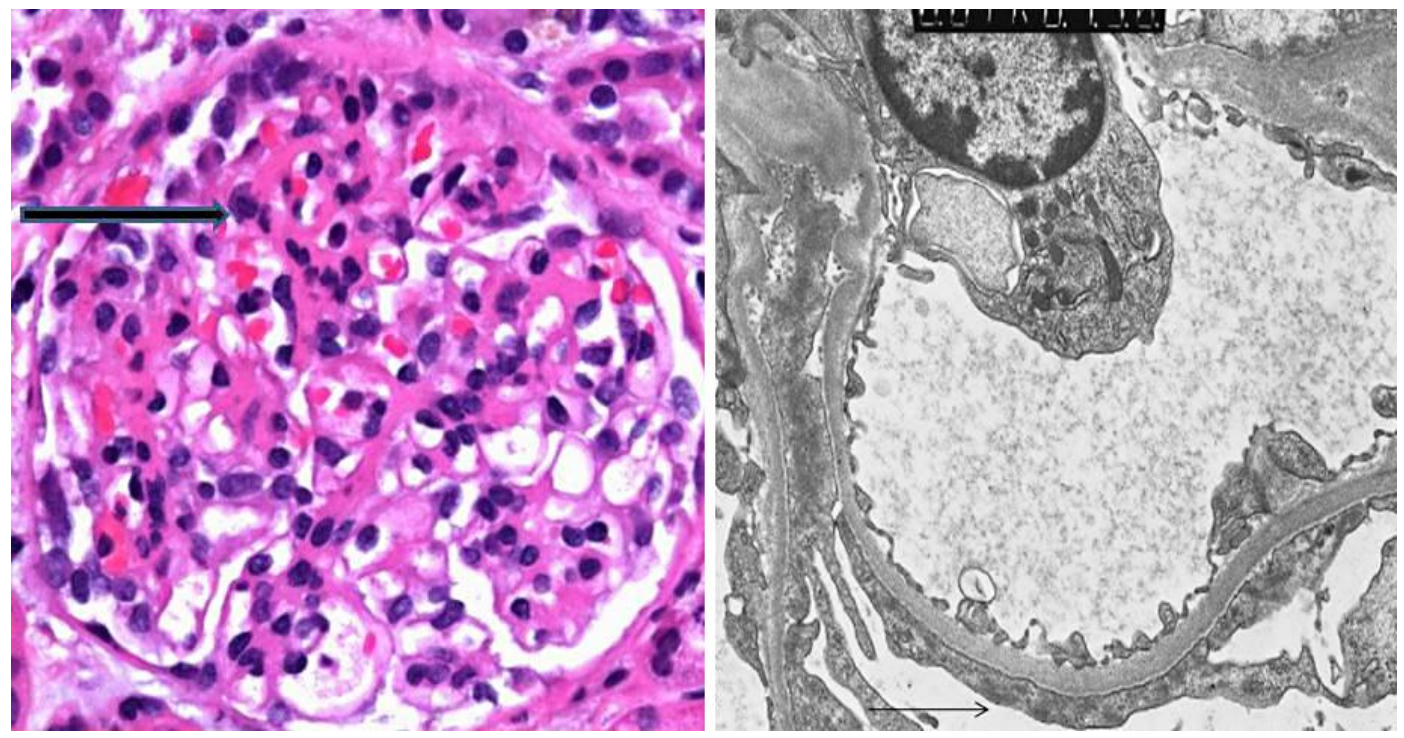

Fig. 1. Light microscopy with PAS stain showing focal areas of sclerosis (arrow) and EM with diffuse foot processes effusion (arrow).
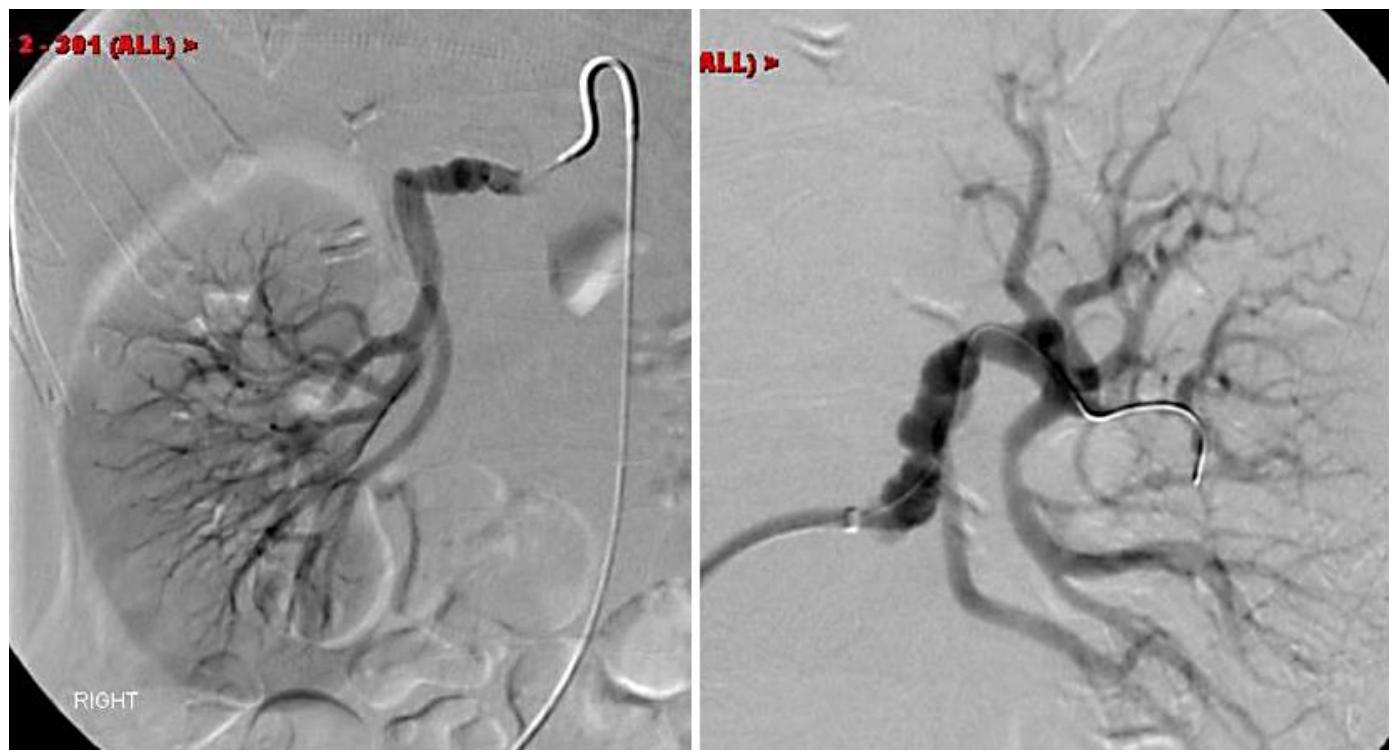

Fig. 2. Right and left renal arteriogram images prior to angioplasty. 


\section{References}

1 Ubara Y, Hara S, Katori H, Yamada A, Morii H: Renovascular hypertension may cause nephrotic range proteinuria and focal glomerulosclerosis in contralateral kidney. Clin Nephrol 1997;48:220-223.

-2 Alkhunaizi AM, Chapman A: Renal artery stenosis and unilateral focal and segmental glomerulosclerosis. Am J Kidney Dis 1997;29:936-941.

-3 Bhowmik D, Dash SC, Jain D, Agarwal SK, Tiwan SC, Dinda AK: Renal artery stenosis and focal and segmental glomerulosclerosis in the contralateral kidney. Nephrol Dial Transplant 1998;13:1562-1564.

4 Alchi B, Shirasaki A, Narita I, et al: Renovascular hypertension: a unique cause of uniateral focal segmental glomerulosclerosis. Hypertens Res 2006;29:203-207.

5 Almirall J, Mendez I, Comet R, Andreu X: Nephrotic syndrome after renal percutaneous transluminal angiplasty. Nephrol Dial Transplant 2000;15:1696-1699.

6 Gephardt GN, Tubbs RR, Novick AC, McMahon JT, Pohl MA: Renal artery stenosis, nephrotic-range proteinuria and focal and segmental glomerulosclerosis. Cleve Clin Q 1984;51:371-376.

7 Kumar A, Shapiro AP: Proteinuria and nephrotic syndrome induced by renin in patients with renal artery stenosis. Arch Intern Med 1980;140:1631-1634.

$>8$ Eiser AR, Katz SM, Swartz C: Reversible nephrotic range proteinuria with renal artery stenosis: a clinical example of renin-associated proteinuria. Nephron 1982;30:374-377.

-9 Thadhani R, Pascual M, Nickeleit V, Tolkoff-Rubin N, Colvin R: Preliminary description of focal and segmental glomerulosclerosis in patients with renovascular disease. Lancet 1996;347:231-233.

10 Bali HK, Jha V: Nephrotic syndrome and recurrent pulmonary edema in bilateral atherosclerotic renal artery stenosis: resolution following angioplasty and stenting. Natl Med J India 2006;19:253-254.

11 Jardine DL, Pidgeon GB, Bailey RR: Renal artery stenosis as a cause of heavy albuminuria. NZ Med J 1993;106:30-31.

12 Ben-Chitrit S, Korzets Z, Podjarny E, Bernheim J: Reversal of nephrotic syndrome due to renovascular hypertension by successful percutaneous angioplasty and stenting. Nephrol Dial Transplant 1995;10:1460-1461.

13 Wolf G: Molecular mechanisms of angiotensin II in the kidney: emerging role in the progression of renal disease: beyond haemodynamics. Nephrol Dial Transplant 1998;13:1131-1142.

14 Bohrer MP, Deen WM, Robertson CR, Brenner BM: Mechanism of angiotensin II-induced proteinuria in the rat. Am J Physiol 1977;233:F13-F21.

$\checkmark 15$ Diez-Sampedro A, Lenz O, Foroni A: Podocytopathy in diabetes: a metabolic and endocrine disorder. Am J Kidney Dis 2011;58:637-646.

16 Rossignol P, Chatellier G, Azizi M, Plouin PF: Proteinuria in renal artery occlusion is related to active renin concentration and contralateral kidney size. J Hypertens 2002;20:139-144.

$\checkmark 17$ D'Agati VD: The spectrum of focal and segmental glomerulosclerosis: new insights. Curr Opin Nephrol Hypertens 2008;17:271-281.

18 Hodgin JB, Rasoulpour M, Markowitz GS, D’Agati VD: Very low birth weight is a risk factor for secondary focal segmental glomerulosclerosis. Clin J Am Soc Nephrol 2009;4:10-13.

19 Sathiapalan RK, Velez MC, McWhorter ME, et al: Focal segmental glomerulosclerosis in children with acute lymphocytic leukemia: case reports and review of literature. J Pediatr Hematol Oncol 1998;20:482-485.

20 Kambham N, Markowitz GS, Valeri AM, et al: Obesity-related glomerulopathy: an emerging epidemic. Kidney Int 2001;59:1498-1509.

-21 Kaufman L, Collins SE, Klotman PE: The pathogenesis of HIV-associated nephropathy. Adv Chronic Kidney Dis 2010;17:36-43.

-22 Khaira A, Upadhyay BK, Sharma A, et al: Hepatitis B virus associated focal and segmental glomerular sclerosis: report of two cases and review of literature. Clin Exp Nephrol 2009;13:373-377.

23 Markowitz GS, Appel GB, Fine PL, et al: Collapsing focal segmental glomerulosclerosis following treatment with high dose pamidronate. J Am Soc Nephrol 2001;12:1164-1172.

-24 Herlitz LC, Markowitz GS, Farris AB, et al: Development of focal segmental glomerulosclerosis after anabolic steroid abuse. J Am Soc Nephrol 2010;21:163-172. 